\title{
Motivation effects in a dichotic listening task as evident from functional magnetic resonance imaging in human subjects
}

\author{
Rolf Larisch ${ }^{a, *}$, Rolf Kötter ${ }^{b}$, Frank Kehren ${ }^{c}$, Marco Tosch $^{a}$, Nadim Joni Shah ${ }^{c}$, \\ Karl Theodor Kalveram ${ }^{d}$, Lutz Jäncke ${ }^{c,}$, , Hans-Wilhelm Müller-Gärtner ${ }^{a}$ \\ ${ }^{a}$ Department of Nuclear Medicine, Heinrich-Heine-University, Düsseldorf, Germany \\ ${ }^{b}$ Center for Anatomy and Brain Research, Heinrich-Heine-University, Düsseldorf, Germany \\ ${ }^{c}$ Institute of Medicine, Research Center Jülich, Jülich, Germany \\ ${ }^{d}$ Department of Psychology, Heinrich-Heine-University, Düsseldorf, Germany \\ e Institute of General Psychology, Otto-von-Guericke-University, Magdeburg, Germany
}

Received 8 February 1999; received in revised form 26 March 1999; accepted 30 March 1999

\begin{abstract}
The present study addresses the effect of motivation on cerebral activity using functional magnetic resonance imaging. Five healthy volunteers performed a dichotic listening task in two sets of three trials during which high or low levels of achievement motivation were introduced. They were told that the first set would be used for calculation of intellectual capacity (high achievement motivation) and the second set for scanner calibration (neutral motivation). In three volunteers, high compared with neutral motivation produced activation in the right prefrontal cortex and the dorsal cingulate. We conclude that motivational effects may lead to significant activations and should be controlled in future cognitive imaging studies. We present preliminary evidence that right prefrontal and dorsal cingulate regions might be involved in motivational processes. (C) 1999 Published by Elsevier Science Ltd. All rights reserved.
\end{abstract}

Keywords: Motivation; Cognitive imaging; Dichotic listening; Magnetic resonance imaging; Activation study; Human study

The study of motivation is the search for principles that aid the understanding of why people and animals choose, initiate, or persist in specific actions. Environmental events may elicit actions directly, as in reflexive attention to an unexpected stimulus. Other actions have been strengthened because they have been reinforced by past events. Relevant neuronal structures for such reinforcement learning were identified as early as 1954 [8]. It appeared that electrical stimulation of brain regions adjacent to the medial forebrain bundle and the lateral hypothalamus is rewarding [6] suggesting that these structures are relevant for motivational processes. More recently, the dopaminergic efferents from substantia nigra to striatum have been examined. Monkeys trained to perform a spatial delayed response task showed phasic responses of dopaminergic neurons to reward [13] suggesting that these fibres are also involved in motivational processes.

Functional magnetic resonance imaging (fMRI) has

* Corresponding author. Tel.: +49-211-811-8540; fax: +49-211811-7041.

E-mail address: rolf.Iarisch@uni-duesseldorf.de (R. Larisch) become widespread for in vivo studying of sensory and cognitive functions. Increased blood flow and oxygenation at the capillary venous level due to brain activity leads to an increasing nuclear magnetic resonance signal [7]. Apart from the cognitive function of interest, additional brain processes might occur simultaneously in most fMRI experiments. Motivational effects are an obvious example. A high motivational state can influence experience and thus affect cerebral activity as measured using fMRI. Therefore, we tested the effect of motivation on the fMRI signal during an auditory discrimination task with high and neutral motivational conditions.

Two types of motivational behaviour have been described: success-oriented subjects perform tasks better when a reward is promised. Failure-oriented subjects perform better when a penalty is imposed. To determine the kind of motivational behaviour we used Schmalt's questionnaire [12], in which volunteers had to assess chances for success and risks in common social situations. Only success-oriented subjects were included in this study. These were five volunteers (two female, three male) aged between 20 and 28 years. All participants were right-handed 


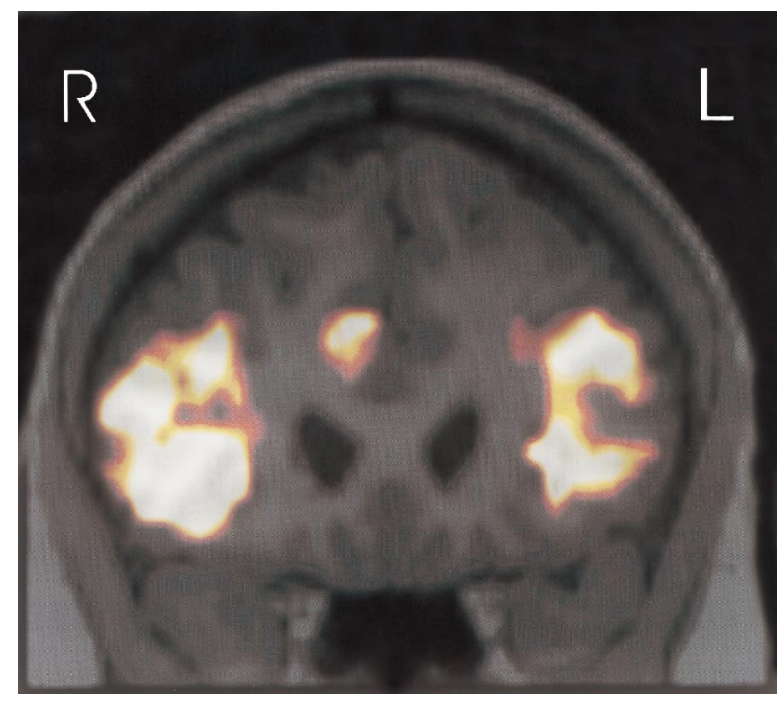

Fig. 1. fMRI signals obtained under baseline condition (syllable presentation) contrasting with the activation condition (ambient scanner noise). Combined analysis of all five subjects superimposed on a structural coronal MRI scan. Activation of the superior temporal gyrus (BA 22) of both hemispheres and of the right anterior cingulate $(P<0.0001)$.

as determined using Annett's handedness questionnaire [1]. Neurological, audiological and psychiatric disorders were excluded by history and physical examination. The study protocol was reviewed and approved by the local Ethics Committee.

All subjects were instructed simultaneously by one of the authors (R.K.) before the fMRI experiment and were asked to perform three competitive trials, which, we told them, would be used for calculation of mental capacity and three similar trials which would be used for scanner calibration. Subjects were told that the task was taken from an intelligence test, which had been shown to be capable of differentiating between professionally successful and unsuccessful people. As further motivation, subjects knew beforehand that the subject with the highest syllable identification score would receive DM $350(\sim 200)$. Actually, the sequence of 'competition' tasks (high motivation) and 'calibration' tasks (neutral motivation) was randomized across each subject. Immediately before each trial, subjects were

Table 1

Significantly activated brain regions due to motivation in three subjects $^{a}$

\begin{tabular}{llllll}
\hline Subject & \multicolumn{2}{l}{$\begin{array}{l}\text { Increases of signal } \\
\text { intensity }\end{array}$} & \multicolumn{2}{l}{$\begin{array}{l}\text { Decreases of signal } \\
\text { intensity }\end{array}$} \\
\cline { 2 - 3 } \cline { 5 - 6 } & Right & Left & & Right & Left \\
\hline A & $5,46,47,9$ & 40,9 & 46 & \\
B & & 31 & & & \\
C & 19,37 & 40,4, hip. & & $10,46,45,21$ & 44,45 \\
\hline
\end{tabular}

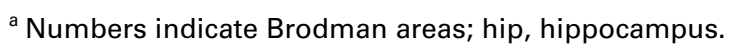

instructed as to perform next, depending on the kind of trial. For this, we suggested that the 'competition' trials should be performed with maximum effort, whereas the 'calibration' trials should be done seriously but would not be used for calculation of the results. We suggested to use them to gain experience for the next competitive task rather than to waste unnecessary effort.

Functional MR images were acquired using a 1.5 Tesla Siemens MRI system (SIEMENS Magnetom Vision, Erlangen, FRG), equipped with echo planar imaging (EPI) capability and a standard radio-frequency head coil. Sequence parameters were described in detail elsewhere [5]. For each experimental condition, a set of 51 images was acquired during multiple alternating periods of 'baseline' (OFF) and 'activation' (ON) conditions. During the ON condition, two syllables out of six (/ka/, /ta/, /pa/, /ga/, $/ \mathrm{da} / \mathrm{or} / \mathrm{ba} /$ ) were presented binaurally and simultaneously with a frequency of $1 \mathrm{~Hz}$. Target syllable was /da/ which occurred with a probability of 0.33 . Subjects were instructed to respond to any occurrence of this target by briefly lifting the index finger of their right hand. During the OFF condition subjects heard only the ambient machine noise. Each series began with nine baseline images (45-s interval), the first three of which were discarded to allow the signal to reach a steady-state, followed by 42 images during which $\mathrm{ON}$ and OFF condition alternated every $30 \mathrm{~s}$. The total duration of each image series was about 4 min.

Image analysis was performed using SPM96 (Statistical Parametric Mapping) software. The alternating ON and OFF periods were modelled using a delayed box-car reference vector. Significantly activated voxels were searched for by using the 'General Linear Model' approach [4]. For this we defined a design matrix comprising contrasts testing for significant activations during auditory stimulation and for differential activations under high and neutral motivation respectively. The $z$-value was set at 3.01 as height threshold. The activated voxels surviving this procedure were additionally subjected to a cluster analysis of spatial extent. Only clusters with a probability of $P<0.05$ (corrected for multiple comparisons) were considered for further evaluation.

Contrasting fMRI signals between auditory OFF and ON conditions revealed significantly activated voxels mainly in the superior temporal gyrus (BA 22) of both hemispheres in all five subjects $(P<0.0001)$. An additionally activated region was located in the right anterior cingulate (Fig. 1). High compared with neutral motivation had no effect in two subjects but significant motivational effects were seen in the other subjects as presented in Table 1. A combined analysis of those three subjects showed increases of signal intensity in right BA 45 and in posterior cingulate (BA 23; Fig. 2). Decreases of signal intensity were seen in right BA 46. In order to quantify the individual performance of the each subject, we analyzed the finger movements separated according to high and neutral conditions. Analysis of performance revealed that subjects performed slightly better under 


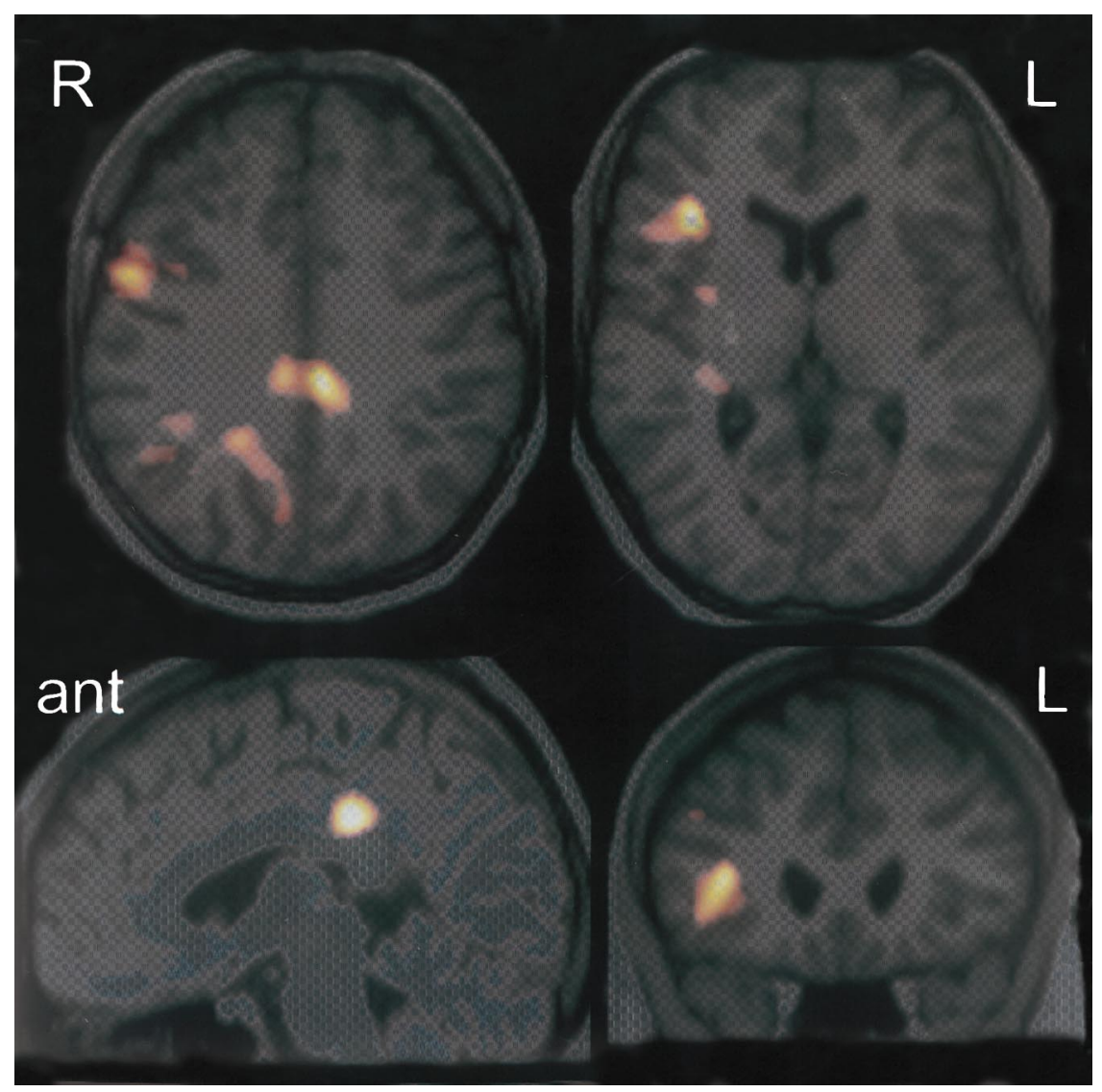

Fig. 2. Effect of motivation on the fMRI signal. A combined analysis shows increases of signal intensity in right BA 45 and in left posterior cingulate area 23. The upper row shows transversal slices at the level of the centrum semiovale and the AC-PC line. A left paramedian sagittal and a coronal slice are displayed in the bottom row.

high $(55.5 \pm 13.6 \%$ correct responses; mean $\pm \mathrm{SD})$ than under neutral motivation $(52.2 \pm 12.4$ correct responses; $P=0.063$; paired $t$-test).

During the auditory phoneme discrimination task we observed activation in the primary and secondary auditory cortex, consistent with the findings of previous studies $[2,9,11]$. We further observed a signal enhancement in the right anterior cingulate. In this context, this activation might indicate subject awareness whilst awaiting syllable presentation and appropriate preparation for the discrimination task [10]. However, with regard to our primary aim of studying motivational processes, the main finding of this work is that three out of five subjects showed significant activation during the high motivation condition. Posterior cingulate and right prefrontal remained significant in the group analysis. Since there was a trend towards better performance under high motivation, these results might partly be related to a difference in group performance. However, activations were not related to auditory cortex. Therefore, it is most likely that they are not due to perceptual functions (i.e. language or auditory perception) but rather to non-perceptual functions, e.g. motivation.

We believe that motivational effects should not be neglected in further cognitive imaging studies. However, since there is currently no psychological instrument available that precisely measures the difference in motivation involved between two tasks, it is difficult to assess the influence of motivational effects on the data. In any case, the recruitment of subjects should involve questionnaires similar to the ones used in this study, ensuring that all subjects are success-oriented or failure-oriented. This helps to build up a population of volunteers who were as far as possible homogeneous regarding their motivated behaviour. When designing the experiment, it should be considered that the control condition involves motivational behaviour that is comparable with the experimental condition. Otherwise, motivational effects should be taken into account seriously, as possible explanations for cortical activations when interpreting the data. In particular, activation of the right dorsolateral prefrontal cortex, which is frequently found in cognitive imaging studies, might also be a consequence of motivational effects.

An open question of this study is why the observed activations due to high motivation were not present in all five subjects although all of them performed as well as possible. Generally, in cognitive imaging studies using a subtraction paradigm, activations of the function under investigation might remain unnoticed when present in both conditions. 
This could also apply to our data in two different ways: subjects who showed similar cerebral activation in the high and neutral motivation condition did in fact perform both tasks with maximum or minimum effort respectively. There is currently no psychological instrument to quantify the difference of motivational effort between different actions. There may also be statistical reasons for our negative findings. Single subject analysis is much more conservative than group analysis. Thus, it is likely that the activation is non-significant in single-subject analysis although they point toward the same direction as the mean activation. The current state of research does not allow us to differentiate between these three possible explanations of our results.

The right dorsolateral prefrontal cortex is involved in multiple cognitive processes. Among them is working memory and especially its central executive system [3]. The role of the posterior cingulate is not entirely clear. According to Vogt [14], posterior cingulate neurons subserve evaluative functions, like monitoring eye movements and responding to sensory stimuli. However, our results have to be confirmed in a larger population before conclusions about the neurobiological basis of motivation can be drawn.

This work was supported by a grant from the 'Deutsche Forschungsgemeinschaft' DFG Ja 737/5-1 awarded to L.J.

[1] Annett, M., Left, Right, Hand and Brain: The Right Shift Theory, Erlbaum, London, 1970.

[2] Binder, J.R., Rao, S.M., Hammeke, T.A., Yetkin, F.Z., Jesmanovicz, A., Bandettini, P.A., Wong, E.C., Estkowski, L.D., Goldstein, M.D., Haughton, V.M. and Hyde, J.S., Functional magnetic resonance imaging of human auditory cortex. Ann. Neurol., 35 (1994) 662-672.

[3] D’Esposito, M., Detre, J.A., Alsop, D.C., Shin, R.K., Atlas, S. and Grossmann, M., The neural basis of the central executive system of working memory. Nature, 378 (1995) 279281.

[4] Friston, K.J., Holmes, A.P., Worsley, K.J., Poline, J.P., Frith, C.D. and Frackowiak, R.S.J., Statistical parametric maps in functional imaging: a general linear approach. Hum. Brain Mapping, 2 (1995) 189-210.

[5] Jäncke, L., Shah, N.J., Posse, S., Grosse-Ryuken, M. and Mueller-Gaertner, H.W., Intensity coding of auditory stimuli: an fMRI study. Neuropsychologia, 36 (1998) 875883.

[6] Mogenson, G. and Phillips, A., Brain-stimulation reward after twenty-five years. Can. J. Psychol., 32 (1978) 54-57.

[7] Ogawa, S., Lee, T.M., Kay, A.R. and Tank, D.W., Brain magnetic resonance imaging with contrast dependent on blood oxygenation. Proc. Natl. Acad. Sci. USA, 87 (1990) 9868-9872.

[8] Olds, J. and Milner, P., Positive reinforcement produced by electrical stimulation of septal area and other regions of the rat brain. J. Comp. Physiol. Psychol., 47 (1954) 419-427.

[9] Petersen, S.E., Fox, P.T., Posner, M.I., Mintun, M.A. and Raichle, M.E., Positron emission tomographic studies of the cortical anatomy of single-word processing. Nature, 331 (1988) 585-589.

[10] Posner, M.I., Attention: the mechanisms of consciousness. Proc. Natl. Acad. Sci. USA, 91 (1994) 7398-7403.

[11] Price, C., Wise, R., Ramsay, S., Friston, K.J., Howard, D., Patterson, K. and Frackowiak, R.S.J., Regional response differences within the human auditory cortex when listening to words. Neurosci. Lett., 146 (1992) 179-182.

[12] Schmalt, H.D., Die Messung des Leistungsmotivs, Hogrefe, Göttingen, 1976.

[13] Schultz, W., Apicella, P. and Ljungberg, T., Responses of monkey dopamine neurons to reward and conditioned stimuli during successive steps of learning a delayed response task. J. Neurosci., 13 (1993) 900-913.

[14] Vogt, B.A., Finch, D.M. and Olson, C.R., Functional heterogeneity in cingulate cortex: the anterior executive and posterior evaluative regions. Cereb. Cortex, 2 (1992) 435443. 